\title{
Expression of the Annexin A1 gene is associated with suppression of growth, invasion and metastasis of nasopharyngeal carcinoma
}

\author{
AIFENG LIU ${ }^{1,2^{*}}$, WEIGUO HUANG ${ }^{1 *}$, GUQING ZENG $^{3}$, XIAOHUA MA $^{4}$, XIAO ZHOU $^{5}$, \\ YAFEI WANG ${ }^{1}$, CHENJIE OUYANG ${ }^{1}$ and AILAN CHENG ${ }^{1}$ \\ ${ }^{1}$ Cancer Research Institute University of South China, Hengyang, Hunan 421001; ${ }^{2}$ Department of Pathology, \\ Changsha Central Hospital, Changsha, Hunan 410004; ${ }^{3}$ School of Nursing, University of South China, Hengyang, \\ Hunan 421001; ${ }^{4}$ Department of Clinical Laboratory, Changsha Central Hospital, Changsha, Hunan 410004; \\ ${ }^{5}$ Department of Pathology, The First People's Hospital of Yueyang, Yueyang, Hunan 414000, P.R. China
}

Received October 29, 2013; Accepted June 17, 2014

DOI: $10.3892 / \mathrm{mmr} .2014 .2656$

\begin{abstract}
Nasopharyngeal carcinoma (NPC) has a highly increased incidence rate $(20 / 100,000)$ in Southern regions of China, while being rare in the rest of the world. NPC is a malignant type of cancer due to its high occurrence rate of metastasis; however, biomarkers for effective diagnosis and treatment are yet to be identified. Annexin A1 is a glucocorticoid-regulated member of a large superfamily of calcium and phospholipid-binding proteins and has been shown to have important roles in tumor development and progression, and was demonstrated to be a prognostic biomarker for head and neck cancer types. A previous study by our group showed that Annexin A1 was decreased in NPC tissue as compared with normal adjacent tissue. To investigate whether Annexin A1 is a potential biomarker for NPC, the present study assessed the effect of the Annexin A1 on the biological behavior (i.e., invasion and metastasis) of the highly metastatic NPC cell line $5-8 \mathrm{~F}$ and the non-metastatic NPC cell line 6-10B. The expression levels of Annexin A1 in the above two cell lines were determined by western blot analysis. Next, the recombinant plasmid pEGFP-C1-Annexin A1 and the small interfering (si) RNA plasmid pRNAT-U6.1-Annexin A1 were used and stably transfected into $5-8 \mathrm{~F}$ and $6-10 \mathrm{~B}$ cells, respectively. These established recombinant cell lines were then used to study the up- and downregulation of Annexin A1, respectively. The correlation of Annexin A1 expression levels with the biological behavior of NPC cell lines was analyzed using a cell proliferation assay, flow cytometry, soft agar colony formation assay, as well as Transwell invasion and migration assays. The results
\end{abstract}

Correspondence to: Professor Ailan Cheng, Cancer Research Institute, University of South China, 28 Changsheng Road, Hengyang, Hunan 421001, P.R. China

E-mail: ailan_cheng@hotmail.com

*Contributed equally

Key words: Annexin A1, invasion, metastasis, nasopharyngeal carcinoma demonstrated that upregulation of Annexin A1 suppressed the proliferation, invasion and migration of NPC cells, while downregulation of Annexin A1 promoted the proliferation, invasion and migration of NPC cells. These findings suggested that Annexin A1 may be a potential biomarker for the development and prognosis of NPC, and its dysregulation may have an important role in its underlying pathogenesis.

\section{Introduction}

Nasopharyngeal carcinoma (NPC) is a rare malignancy in the majority of the world, while is has an increased prevalence in China, particularly in the Southern regions of the country $(1,2)$. The average incidence of NPC worldwide is $<1 / 100,000$ population (3). However, in endemic areas of China, the incidence has reached 20/100,000 (2,4,5). Approximately 70-80\% of patients with NPC present with lymph node metastases in the neck (6), and $\sim 4.2 \%$ of these patients present with distant metastasis to the bone, lung, liver and central nervous system (7). However, the molecular mechanism of NPC pathogenesis remains elusive and reliable biomarkers of the disease have yet to be discovered. Therefore, it is critical to investigate and identify effective biomarkers that may be used for diagnosis and as potential therapeutic targets of NPC.

Annexin A1, a glucocorticoid-regulated member of a large superfamily of calcium and phospholipid-binding proteins, is widely expressed in numerous different cell types $(8,9)$. Accumulating evidence revealed that Annexin A1 has important roles in tumor development and progression (10-13). For example, in head and neck cancer types, the expression levels of Annexin A1 have been associated with advanced stages of the disease, differentiation status and metastasis (10). It may therefore be an effective differentiation marker for the detection of head and neck squamous cell carcinomas. A previous study by our group demonstrated that the expression levels of Annexin A1 are decreased in NPC as compared with those in normal nasopharyngeal epithelial tissues, using laser capture microdissection and proteomic analysis (14). However, the specific function of Annexin A1 in NPC has remained elusive. Therefore, the present study sought to investigate the role of Annexin A1 in the development and progression of NPC. 
To examine the role of Annexin A1 in NPC, the highly metastatic cell line 5-8F and the non-metastatic cell line 6-10B were selected. Annexin A1 expression in 5-8F and in 6-10B cells was stably up- or downregulated by using plasmids or RNA interference, respectively. Then, the biological behavior of the cells was examined using proliferation assay, soft agar colony formation assay, flow cytometry, as well as Transwell invasion and migration assays, to assess whether Annexin A1 may be a potential molecular biomarker of NPC that may be used in diagnosis and treatment.

\section{Materials and methods}

Materials. The plasmid p enhanced green fluorescent protein (EGFP)-C1 and Annexin A1 small interfering (si)RNA were purchased from RiboBio Co. Ltd. (Guangzhou, China). The plasmid pRNAT-U6.1 was purchased from Biomics Biotechnologies Co. Ltd. (Jiangsu, China). Anti-Annexin A1 monoclonal antibodies were purchased from Sigma-Aldrich (St. Louis, MO, USA). Horseradish peroxidase (HRP)-labeled secondary antibodies [goat anti-rabbit immunoglobulin (Ig)G] and polyvinylidene difluoride (PVDF) membranes were purchased from EMD Millipore (Billerica, MA, USA). Radioimmunoprecipitation assay (RIPA) buffer was purchased from Pierce Biotechnology, Inc. (Rockford, IL, USA). The Transwell chamber and Matrigel were purchased from BD Biosciences (Franklin Lakes, NJ, USA). The Cell Counting kit-8 (CCK-8) was purchased from Dojindo Laboratories (Kumamoto, Japan). LE Agarose was obtained from HydraGene (Xiamen, China). All of the cell culture flasks and plates were purchased from Corning Inc. (Corning, NY, USA).

Cell culture. Human NPC cell lines 5-8F (highly metastatic) and 6-10B (non-metastatic) were kindly donated by the Key Laboratory of Cancer Proteomics of Chinese Ministry of Health of Xiangya Hospital (Changsha, Hunan, China), following purchase from the American Type Culture Collection (Manassas, VA, USA), and grown in RPMI-1640 medium (Invitrogen Life Technologies, Carlsbad, CA, USA) supplemented with $10 \%$ fetal bovine serum (FBS; Invitrogen Life Technologies). The cells were maintained at $37^{\circ} \mathrm{C}$ in a humidified $5 \% \mathrm{CO}_{2}$ atmosphere, and were shown to be free from mycoplasmic contamination.

Western blot. The cells were pelleted by centrifugation at $2,500 \mathrm{x} g$ for $5 \mathrm{~min}$ and then washed twice with ice-cold phosphate-buffered saline (PBS), lysed on ice for $15 \mathrm{~min}$ in $100 \mu 1$ of RIPA buffer and centrifuged at $14,000 \times \mathrm{g}$ for 15 min. The supernatants were collected and the protein concentrations were determined using a Bradford protein assay. Aliquots of cell lysates ( $80 \mu \mathrm{g}$ proteins) were boiled for 5 min in SDS-containing sample buffer and were separated using SDS-PAGE on a $12 \%$ polyacrylamide gel. The proteins were transferred to a PVDF membrane and western blot analyses were performed with the indicated antibody according to the manufacturer's instructions. The proteins were detected using an enhanced chemiluminescence western blot system (Thermo Fisher Scientific, Inc., Waltham, MA, USA) and the band intensity was measured using densitometry analyzed with ImageJ software (National Institutes of Health, Bethesda, MD, USA).

Transient transfection with siRNAs. Three 21-nucleotide siRNA duplexes targeting different coding regions of Annexin A1 (GenBank accession no. NM_000700) were designed and chemically synthesized by RiboBio Co. Ltd. (Guangzhou, China). The secondary structures of target mRNA was predicted using RNAstructure 3.7 software. The siRNA nucleotide sequences were as follows: siRNA1, sense 5'-GGAUGAAACACUUAAGAAAdTdT-3' and antisense 3'-dTdTCCUACUUUGUGAAUUCUUU-5'; siRNA2, sense 5'-GCAGCAUAUCUCCAGGAAAdTdT-3' and antisense 3'-dTdT CGUCGUAUAGAGGUCCUUU-5'; siRNA3, sense 5'-GUUCUGAAAUUGACAUGAA dTdT-3' and antisense 3'-dTdT CAAGACUUUAACUGUACUU-5'. The siRNAs were transfected into 6-10B cells using Lipofectamine 2000 (Invitrogen Life Technologies) according to the manufacturer's instructions and cultured for an additional $48 \mathrm{~h}$. The samples were then prepared for western blot analysis in accordance with the aforementioned methods.

Recombinant plasmid construction. Recombinant plasmids were constructed in several steps as follows: i) Overexpression plasmid: Primers of Annexin A1 were designed according to gene sequence information of the coding region. The Annexin A1 cDNA isolated from HEK-293 cells was used as the template for polymerase chain reaction (PCR). The product was then cloned into the eukaryotic expression vector pEGFP-C1, between the XhoI and BanHI restriction sites. The positive clones were identified using restriction endonuclease digestion and subsequently sequenced by Sangon Biotech Co., Ltd. (Shanghai, China); ii) Interference plasmids: The siRNA3 fragment was regarded as the effective sequence based on the siRNA-screening assay as described above. Appropriate oligos were designed and used to generate the PCR fragment that was ligated into the BamHI and HindIII restriction sites of the siRNA vector, pRNAT-U6.1. Positive clones were identified by restriction endonuclease digestion, and subsequently sent for sequencing to Sangon Biotech Co., Ltd.

Stable transfection. Highly metastatic 5-8F NPC cells were transfected with pEGFP-C1 or pEGFP-C1-Annexin A1 and non-metastatic 6-10B NPC cells were transfected with pRNAT-U6.1-siRNA-negative or pRNAT-U6.1-siRNA-Annexin A1 using Lipofectamine 2000 Reagent (Invitrogen Life Technologies), according to the manufacturer's instructions. Following 14 days of selection in RPMI-1640 medium containing 500 or $400 \mu \mathrm{g} / \mathrm{ml} \mathrm{G} 418$ (Invitrogen Life Technologies), individual G418-resistant colonies were isolated and expanded. The expression of Annexin A1 in these clones was determined using western blot analysis as described in accordance with the aforementioned methods.

CCK-8 proliferation assay. Growth curves of $5-8 \mathrm{~F}$, 5-8F/pEGFP-C1, 5-8F/pEGFP-C1-Annexin A1, 6-10B, 6-10B/siRNA-negative and 6-10B/siRNA-Annexin A1 cells were analyzed using the CCK-8 proliferation kit (Dojindo Laboratories). Briefly, the cells were plated in 96-well culture 
A

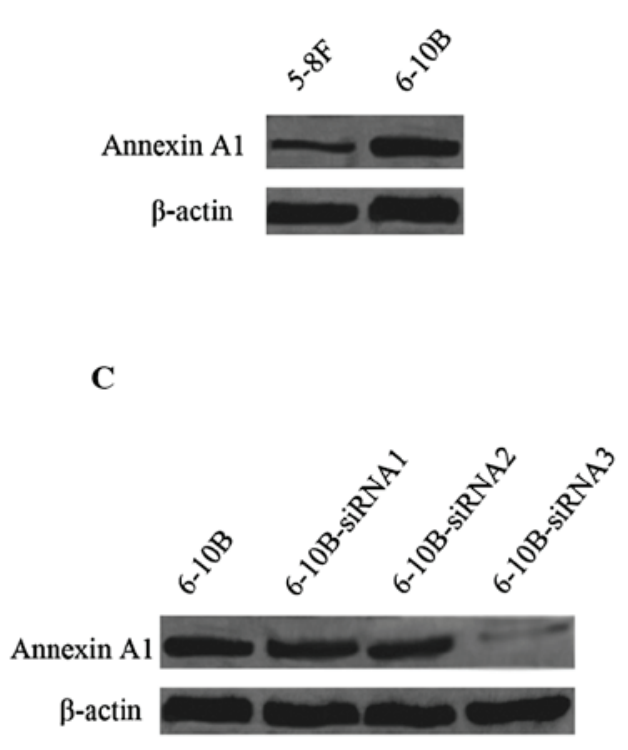

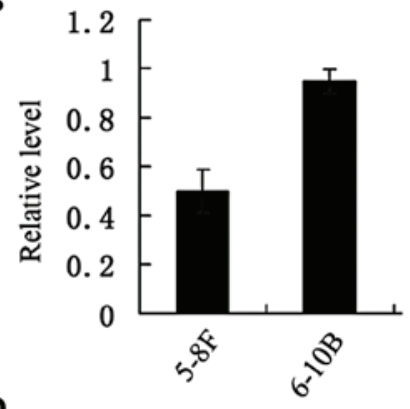

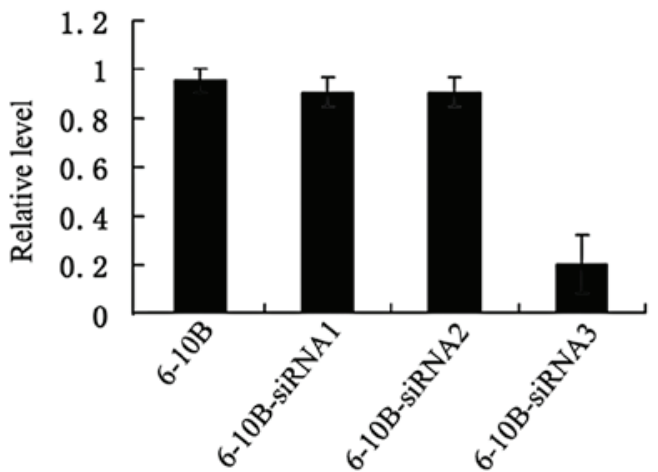

Figure 1. Expression of Annexin A1 in nasopharyngeal carcinoma cell lines. (A) Western blot analysis demonstrates the expression levels of Annexin A1 in the 5-8F and 6-10B cell lines. (B) Bar chart demonstrating the relative protein expression levels in the 5-8F and 6-10B cell lines. (C) Western blot analysis demonstrating the expression levels of Annexin A1 in 6-10B cells transiently transfected with siRNAs. (D) Bar chart demonstrating the relative protein expression levels in 6-10B cells transiently transfected with siRNAs as determined by densitometric analysis. siRNA, small interfering RNA.

plates at a density of $1 \times 10^{3}$ cells/well. Following 1, 2, 3, 4 and 5 days, a $10 \mu$ l CCK- 8 reaction solution was added, respectively. The optical density was then read at a wavelength of $452 \mathrm{~nm}$ in an ELISA plate reader (Labsystem Multiskan MK3; Thermo Fisher Scientific, Inc.) 3 h later.

Cell cycle analysis. For DNA content analysis, 5-8F, 5-8F/pEGFP-C1, 5-8F/pEGFP-C1-Annexin A1, 6-10B, 6-10B/siRNA-negative and 6-10B/siRNA-Annexin A1 were harvested and washed in ice-cold PBS and centrifuged $(1,000 \mathrm{x} g$ for $5 \mathrm{~min})$, then fixed in ice-cold $70 \%$ ethanol at $-20^{\circ} \mathrm{C}$. Then the samples were analyzed using a FACScan flow cytometer (Beckman Coulter, Miami, FL, USA). The results obtained were analyzed using ModFit (Verity Software House, Topsham, ME, USA).

Soft agar colony formation assay. The effect of Annexin A1 protein levels on the anchorage-independent growth of 5-8F, 5-8F/pEGFP-C1, 5-8F/pEGFP-C1-Annexin A1, 6-10B, 6-10B/siRNA-negative and 6-10B/siRNA-Annexin A1 was examined using a soft agar colony formation assay. Briefly, the culture compartments of six-well plates (Corning Inc.) were filled with $1.5 \mathrm{ml} 0.5 \%$ agar solution in RPMI-1640 medium containing 10\% FBS as the basal agar layer, and $1 \mathrm{ml} 0.3 \%$ agar solution with $1 \times 10^{4}$ NPC cells in RPMI-1640 medium containing $10 \%$ FBS were transferred onto the basal agar layer as the cell agar layer. Cells were then continuously cultured until visible colonies were formed (10 days). The colonies containing $>50$ cells were counted. The rate of colony formation was indicated by the ratio of the number of colonies over the number of seeded cells. The experiments were repeated for three times and a mean value was calculated.

Cell migration and invasion assays. The migration and invasion activity of $5-8 \mathrm{~F}, 5-8 \mathrm{~F} / \mathrm{pEGFP}-\mathrm{C} 1$, 5-8F/pEGFP-C1-Annexin A1, 6-10B, 6-10B/siRNA-negative and $6-10 \mathrm{~B} / \mathrm{siRNA}-\mathrm{Ann}$ exin A1 cells were assayed using a transwell cell culture chamber. The cells were added to the transwell chamber (pore size, $12 \mu \mathrm{m}$; BD Biosciences). The number of cells that migrated through the membrane was determined $24 \mathrm{~h}$ later. For the invasion assays, Matrigel (BD Biosciences) was added to the Transwell chambers and incubated overnight prior to seeding of the cells. The number of membrane-penetrating cells was counted $24 \mathrm{~h}$ later. The migration and the invasion assays were performed in quadruplicate for each cell line tested.

Statistical analysis. Values are presented as the mean \pm standard deviation of the results of at least three experiments. One-way analysis of variance and Student's t-test were used to assess differences in cell growth rate, colony formation, cell cycle distribution, cell migration and invasion. $\mathrm{P}<0.05$ was considered to indicate a statistically significant difference.

\section{Results}

Detection of Annexin A1 levels in 5-8F and 6-10B cells. Western blot analysis was performed to detect the expression levels of Annexin A1 in the two NPC cell lines with different metastatic potentials. As demonstrated in Fig. 1, when 

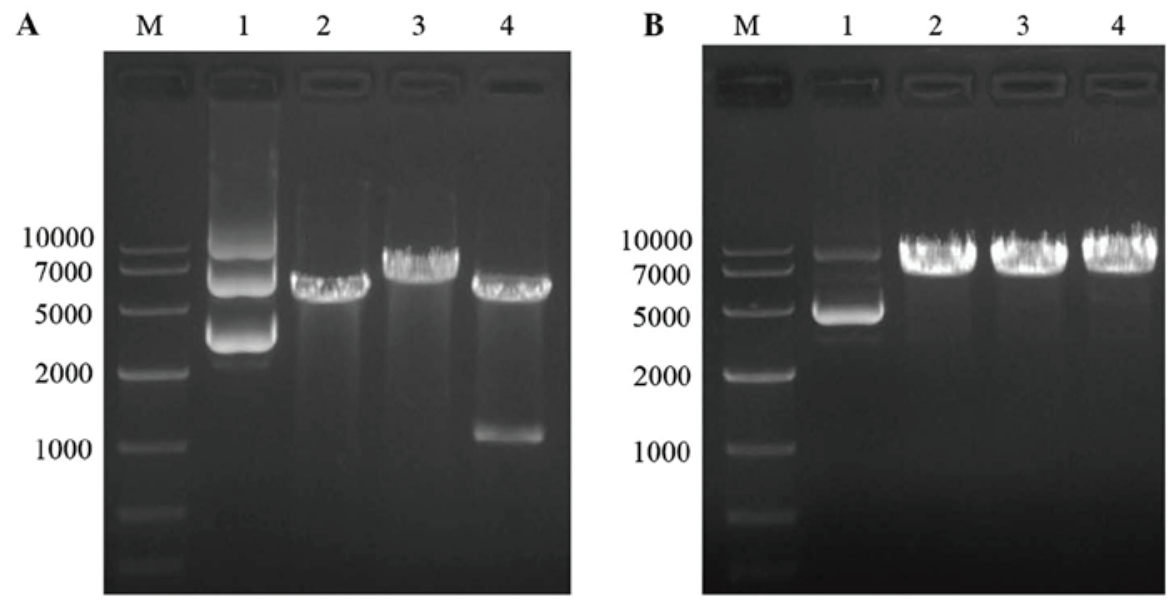

Figure 2. Identification of recombinant Annexin A1 constructs restrict endonuclease digestion. (A) Annexin A1 overexpression recombinant; lane M, DL10000 marker; lane 1, pEGFP-C1; lane 2, pEGFP-C1 digested with BamHI; lane 3, pEGFP-C1-Annexin A1 digested with BamHI; lane 4, pEGFP-C1-Annexin A1 with BamHI and XhoI. (B) Annexin A1 interference recombinant; lane M, DL10000 marker; lane 1, pRNAT-U6.1; lane 2, pRNAT-U6.1-siRNA-negative digested with BamHI; lane 3, pRNAT-U6.1-siRNA-Annexin A1 digested with BamHI; lane 4, pRNAT-U6.1-Annexin A1 digested with HindIII and BamHI. siRNA, small interfering RNA; EGFP, enhanced green fluorescent protein.

compared with the $5-8 \mathrm{~F}$ cells with high metastatic potential, there was significantly higher expression of Annexin A1 in the 6-10B cells with low metastatic potential $(\mathrm{P}<0.01)$. This result indicated that the protein Annexin A1 may be inversely correlated with the metastatic potential of NPC cell lines.

siRNA-mediated inhibition of the expression of Annexin Al in $6-10 \mathrm{~B}$ cells. The above data demonstrated a high expression of Annexin A1 in 6-10B cells and a low expression in 5-8F cells. In order to identify an effective RNA interference sequence of Annexin A1, three different siRNAs were selected and tested for silencing the expression of Annexin A1 in the 6-10B cell line. Three siRNA duplexes targeting different coding regions of human Annexin A1 mRNA, named siRNA1, siRNA2 and siRNA3, were designed, synthesized and transfected into the NPC cell line 6-10B. The expression levels of Annexin A1 in 6-10B silenced and non-silenced cell lines were assessed at the protein level using western blot analysis. As revealed in Fig. 1, higher Annexin A1 protein levels were detected in the 6-10B cells, with siRNA3 (100 nmol/l) being the most effective in suppressing Annexin A1 expression, while siRNA2 (100 nmol/l) inhibited Annexin A1 expression to a lesser extent. However, transfection of siRNA1 (100 nmol/l) only marginally affected Annexin A1 expression in the 6-10B cells. Therefore, siRNA3 was selected for the subsequent experiments, since it was the most effective inhibitor of Annexin A1 expression.

Identification of recombinant Annexin Al constructs using restriction endonuclease digestion. To verify that the recombinant plasmids were successfully constructed, restriction digestion with XhoI, BamI and HindIII was utilized and agarose gel electrophoresis was conducted to analyze the target fragment. As demonstrated in Fig. 2, the Annexin A1 target fragment was in the expected position (Fig. 2A). However, the inhibitor fragment was not apparent, as the fragment was only $64 \mathrm{bp}$ in length (Fig. 2B). All of the constructs were subsequently verified using sequencing.
Establishment of the stably transfected NPC cell lines. To study the association between the levels of Annexin A1 expression in the NPC cell lines and their biological behavior (i.e., metastatic ability, invasiveness, etc.), the blank plasmids of pEGFP-C1 and recombinant plasmid of pEGFP-C1-Annexin A1 were transfected into the 5-8F cells. Meanwhile, the 6-10B cells were transfected with the plasmids pRNAT-U6.1-siRNA-negative and pRNAT-U6.1-siRNA-Annexin A1. Stable G418-resistant clonal cell lines were obtained. These were then subjected to western blot analysis. As demonstrated in Fig. 3, Annexin A1 expression in 5-8F/pEGFP-C1-Annexin A1 cells was greater than that in $5-8 \mathrm{~F}$ or $5-8 \mathrm{~F} / \mathrm{pEGFP}-\mathrm{C} 1$ cells. By contrast, there was a lower expression of Annexin A1 in $6-10 \mathrm{~B} / \mathrm{siRNA}-\mathrm{Annexin} \mathrm{A} 1$ than in the $6-10 \mathrm{~B}$ or 6-10B/siRNA-negative cell lines. These data demonstrated that the NPC cell lines in which Annexin A1 expression was stably up- or downregulated had been successfully established, and various stable cell lines were selected for subsequent use.

Annexin Al regulates NPC cell growth in vitro. To assess the effect of Annexin A1 on NPC cells, proliferation, colony-forming and cell cycle assays were performed with the two different NPC cell lines and their derivatives. First, the effect of Annexin A1 upregulation or downregulation on NPC cell growth was examined. As revealed in Fig. 4A and B, with increased expression of Annexin A1 in 5-8F cells, the proliferation rate was more significantly decreased than that in the non-transfected and mock-transfected cells $(\mathrm{P}<0.01)$. By contrast, when the expression of Annexin A1 in 6-10B cells was decreased, the proliferation rate was significantly increased compared with that of the non-transfected and control-transfected cells $(\mathrm{P}<0.05)$. The effect of Annexin A1 on cell colony formation was further investigated (Fig. 4C and D). Similarly, as described above, $5-8$ F cells with a higher expression of Annenxin A1 demonstrated a significantly lower colony-forming ability compared with the negative controls. 
Table I. Effect of Annexin A1 expression on the cell cycle of nasopharyngeal carcinoma cells.

\begin{tabular}{|c|c|c|c|}
\hline \multirow[b]{2}{*}{ Groups } & \multicolumn{3}{|c|}{ Percentage of periods $( \pm$ SD) } \\
\hline & G1 & $\mathrm{G} 2$ & $\mathrm{~S}$ \\
\hline $5-8 \mathrm{~F}$ & $66.22 \pm 1.21$ & $5.24 \pm 0.89$ & $28.54 \pm 1.13$ \\
\hline 5-8F/pEGFP-C1 & $66.12 \pm 1.65$ & $5.92 \pm 1.24$ & $27.96 \pm 1.67$ \\
\hline 5-8F/pEGFP-C1-Annexin A1 & $77.93 \pm 2.14^{\mathrm{a}}$ & $13.20 \pm 1.46$ & $8.88 \pm 2.01^{\mathrm{b}}$ \\
\hline $6-10 \mathrm{~B}$ & $65.16 \pm 1.36$ & $14.83 \pm 0.64$ & $20.00 \pm 0.99$ \\
\hline 6-10B/siRNA-negative & $65.39 \pm 1.52$ & $15.17 \pm 0.85$ & $19.44 \pm 1.56$ \\
\hline 6-10B/siRNA-Annexin A1 & $59.11 \pm 1.91$ & $14.33 \pm 1.24$ & $26.56 \pm 1.87^{c}$ \\
\hline
\end{tabular}

${ }^{\mathrm{a}} \mathrm{P}<0.05, \quad{ }^{\mathrm{b}} \mathrm{P}<0.01,5-8 \mathrm{~F} / \mathrm{pEGFP}-\mathrm{C} 1-\mathrm{Ann} e x i n \mathrm{~A} 1$ vs. $5-8 \mathrm{~F}$ or $5-8 \mathrm{~F} / \mathrm{pEGFP}-\mathrm{C} 1 ;{ }^{\mathrm{c}} \mathrm{P}<0.05,6-10 \mathrm{~B} / \mathrm{siRNA}-\mathrm{Annexin} \mathrm{A} 1$ vs. 6-10B or 6-10B/siRNA-negative. siRNA, small interfering RNA; SD, standard deviation; EGFP, enhanced green fluorescent protein.
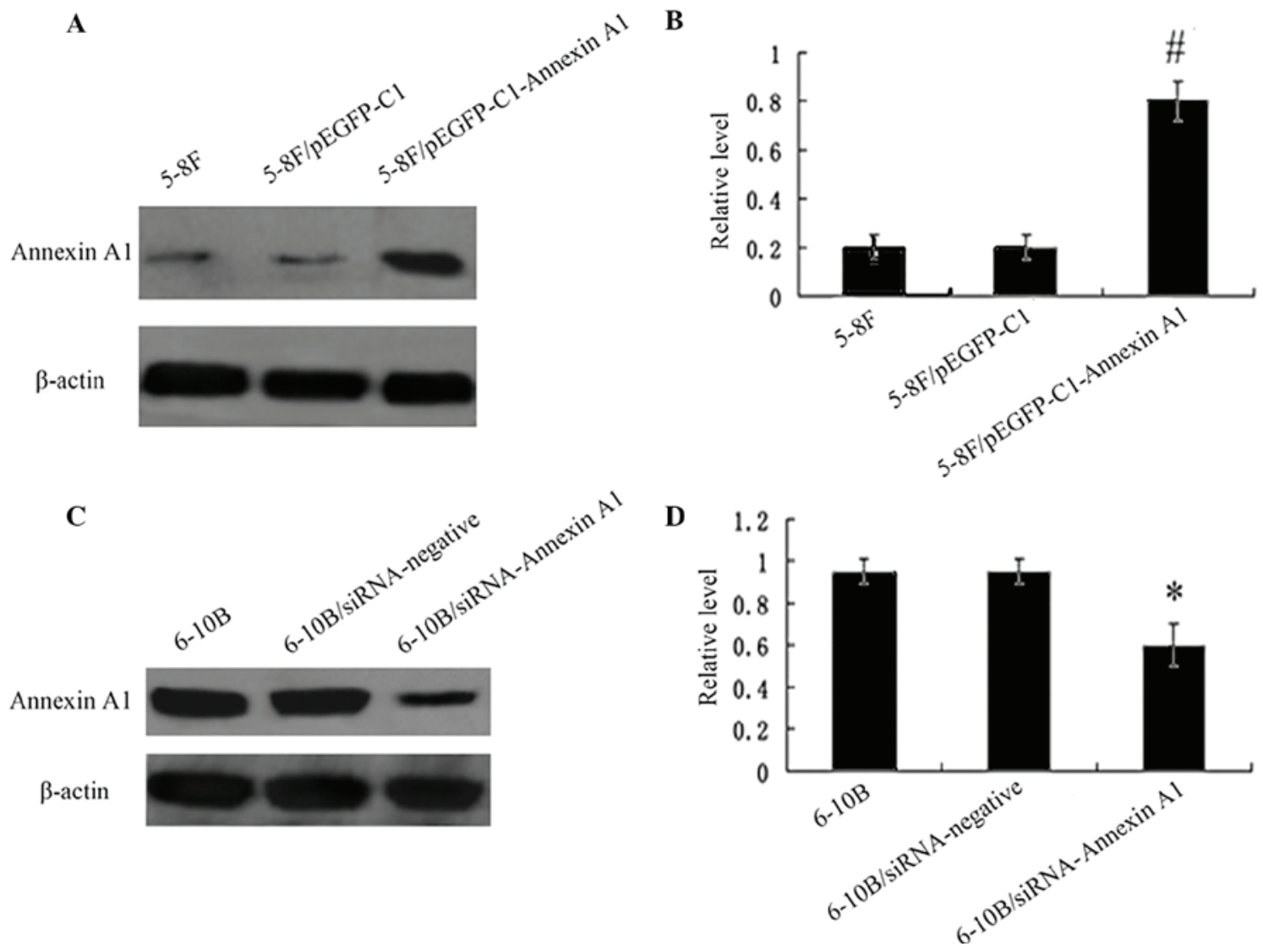

Figure 3. The levels of Annexin A1 expression in groups of 5-8F and 6-10B cell lines. (A) Western blot analysis demonstrates the expression levels of Annexin A1 in pEGFP-C1-Annexin A1-transfected and control pEGFP-C1-transfected 5-8F cells. (B) Bar chart reveals the relative changes in the protein expression levels in the different plasmid-transfected NPC cell lines as determined by densitometric analysis. (C) Western blot analysis of the expression levels of Annexin A1 in pRNAT-U6.1-siRNA-Annexin A1-transfected and control pRNAT-U6.1-siRNA-negative-transfected 6-10B cells. (D) Bar graph demonstrates the relative changes in the protein expression levels in the different siRNAs NPC cell lines as determined by densitometric analysis. Data are presented as the mean \pm standard deviation. ${ }^{*} \mathrm{P}<0.01$ vs. the $5-8 \mathrm{~F}$ and $5-8 \mathrm{~F} / \mathrm{pEGFP}-\mathrm{C} 1$ cells; ${ }^{*} \mathrm{P}<0.05$ vs. the $6-10 \mathrm{~B}$ and $6-10 \mathrm{~B} /$ siRNA-negative cell lines. NPC, nasopharyngeal carcinoma; siRNA, small interfering RNA; EGFP, enhanced green fluorescent protein.

With increased expression of Annexin A1 in 5-8F cells, the number of colonies was lower than that in the control groups. Decreased expression of Annexin A1 in 6-10B cells resulted in enhanced colony-forming activity compared with the two other control cell lines. It is plausible that Annexin A1 suppressed the viability of NPC cells. Then, the cell cycle of those cells was analyzed by flow cytometry (Table I). The results demonstrated that in the 5-8F cells transfected with pEGFP-C1-Annexin A1, the percentage of cells arrested in $\mathrm{S}$ phase was significantly decreased, while the percentage of cells in G1 phase was significantly increased. The opposite was observed in the 6-10B cells, such that silencing Annexin A1 led to an increase in the percentage of cells in $\mathrm{S}$ phase. However, the percentage of cells in G1 phase was significantly decreased. 
A

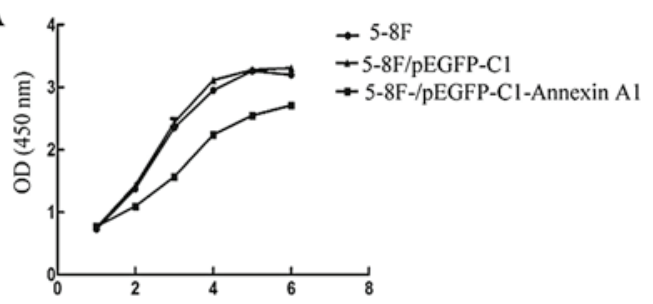

$\mathrm{t} / \mathrm{d}$

C
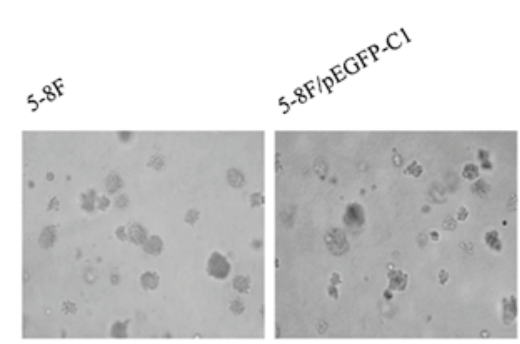

E
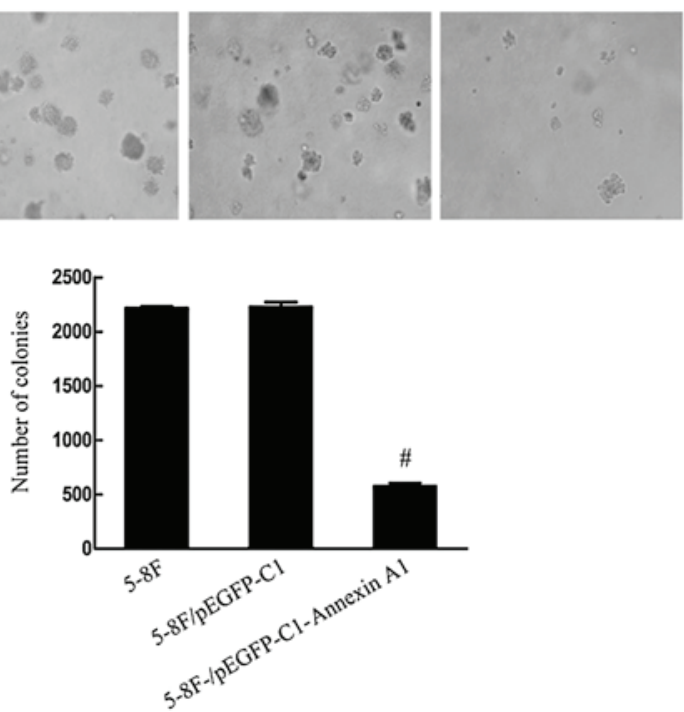

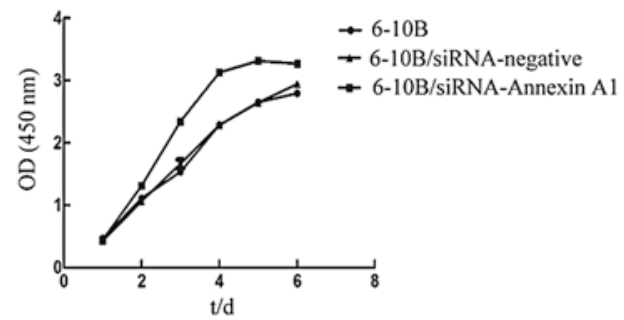

D
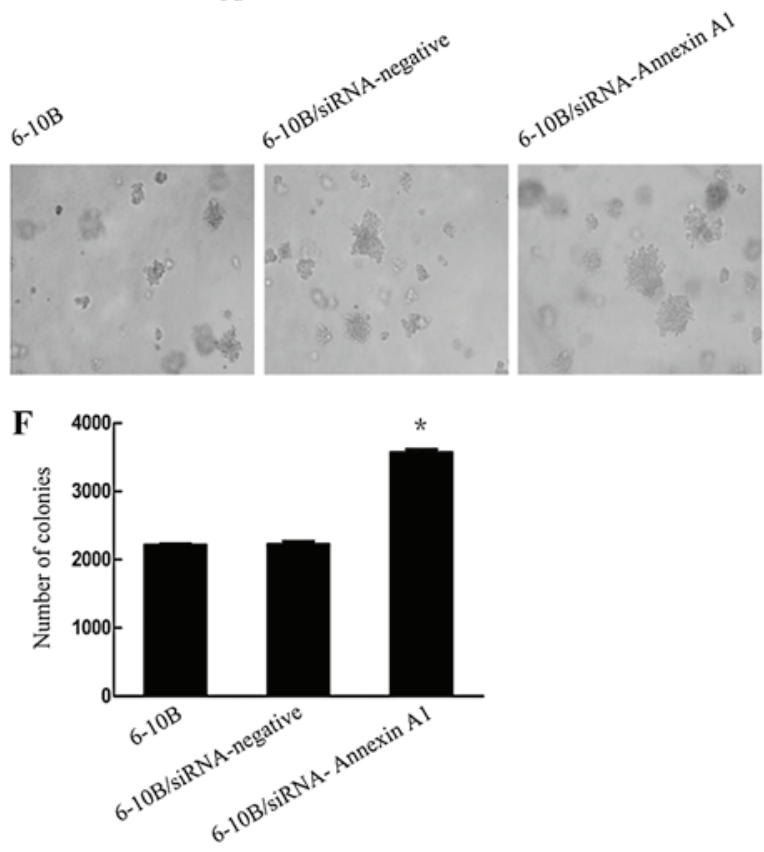

Figure 4. Effect of Annexin A1 expression in NPC cells. (A) Growth curve of 5-8F cells and their transfectants; (B) growth curve of 6-10B cells and their transfectants; (C) in vitro colony formation of 5-8F cells and their transfectants (magnification, x100); (D) in vitro colony formation of 6-10B cells and their transfectants (magnification, x100); (E) bar chart demonstrating the average number of colonies of 5-8F cells and their transfectants per field; (F) bar chart demonstrating the average number of colonies of $6-10 \mathrm{~B}$ cells and their transfectants per field. Bars represent the mean values, error bars represent the upper 95\% confidence intervals. ${ }^{*} \mathrm{P}<0.05,{ }^{\#} \mathrm{P}<0.01$ compared with the empty/negative vector-transfected or untransfected cells. NPC, nasopharyngeal carcinoma; siRNA, small interfering RNA; OD, optical density; EGFP, enhanced green fluorescent protein.

Table II. The numbers of nasopharyngeal carcinoma cells and their transfectants through the transwell chamber.

Number $( \pm$ SD)

\section{Groups}

\section{$5-8 \mathrm{~F}$}

$5-8 \mathrm{~F} / \mathrm{pEGFP}-\mathrm{C} 1$

5-8F/pEGFP-C1-Annexin A1

6-10B

6-10B/siRNA-negative

6-10B/siRNA-Annexin A1
Migratory cells Invasive cells

$\begin{array}{cc}515 \pm 10 & 233 \pm 13 \\ 520 \pm 15 & 225 \pm 15 \\ 203 \pm 25^{\mathrm{a}} & 28 \pm 6^{\mathrm{a}} \\ 55 \pm 9 & 52 \pm 6 \\ 48 \pm 8 & 49 \pm 9 \\ 202 \pm 28^{\mathrm{b}} & 210 \pm 16^{\mathrm{b}}\end{array}$

${ }^{\mathrm{a}} \mathrm{P}<0.01,5-8 \mathrm{~F} / \mathrm{pEGFP}-\mathrm{C} 1-$ Annexin A1 vs. $5-8 \mathrm{~F}$ or $5-8 \mathrm{~F} / \mathrm{pEGFP}-\mathrm{C} 1$; ${ }^{b} \mathrm{P}<0.01,6-10 \mathrm{~B} / \mathrm{siRNA}-\mathrm{Annexin} \mathrm{A} 1$ vs. $6-10 \mathrm{~B}$ or 6-10B/siRNA-negative. siRNA, small interfering RNA; SD, standard deviation; EGFP, enhanced green fluorescent protein.

Effects of Annexin Al on NPC cell motility in vitro. To examine the potential role of Annexin A1 in NPC, Annexin A1 expression in NPC cells was modulated using stable transfection of 5-8F cells with pEGFP-C1-Annexin A1 (or empty expression vector as the control) and 6-10B cells with pRNAT-U6.1-siRNA-Annexin A1 (or pRNAT-U6.1-siRNA-negative as a the control), and then measured the migratory and invasive ability of the parental (i.e. untransfected) 5-8F and 6-10B cells and their stable transfectants using in vitro migration and invasion assays. As demonstrated in Fig. 5 and Table II, the migration of 5-8F cells transfected with pEGFP-C1-Annexin A1 was decreased by $\sim 2.5$-fold compared with the migration of $5-8 \mathrm{~F}$ cells transfected with the control vector $(\mathrm{P}<0.01)$. Conversely, the migration of $6-10 \mathrm{~B}$ cells transfected with pRNAT-U6.1-siRNA-Annexin A1 was 4-fold higher than the migration of 6-10B cells transfected with the control vector $(\mathrm{P}<0.01)$. Similar results were obtained by the invasion assay. The 5-8F cells transfected with pEGFP-C1-Annexin A1 cells were $\sim 9$-fold less invasive than $5-8 \mathrm{~F}$ cells transfected with the control vector $(\mathrm{P}<0.01)$. Conversely, the $6-10 \mathrm{~B}$ cells transfected with pRNAT-U6.1-siRNA-Annexin A1 cells were 4 -fold more invasive than $6-10 \mathrm{~B}$ cells transfected with the control vector $(\mathrm{P}<0.01)$. These results suggested that Annexin A1 expression levels were inversely associated with the invasiveness of NPC cells in vitro. 
A

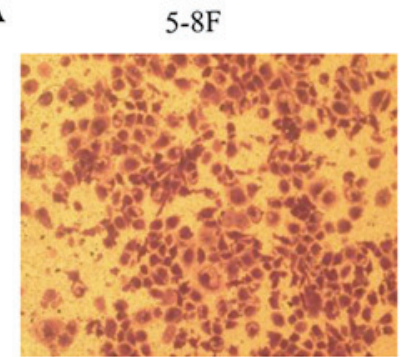

B

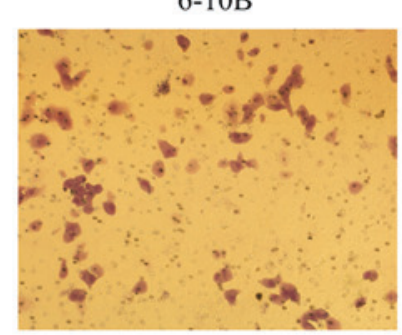

C

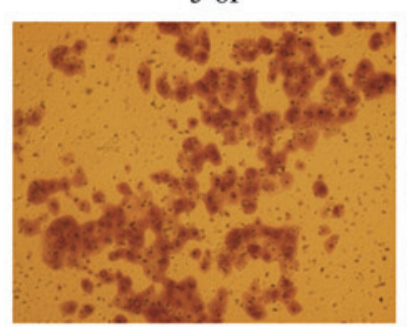

D

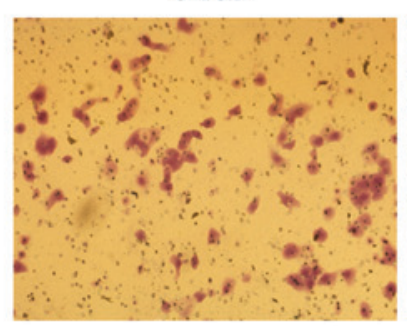

$5-8 \mathrm{~F} / \mathrm{pEGFP}-\mathrm{C} 1$

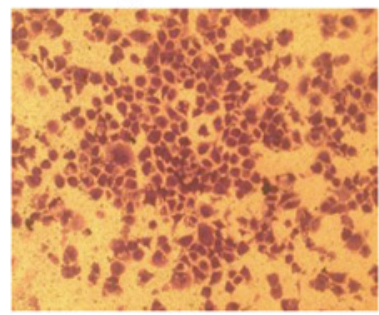

6-10B/siRNA-negative

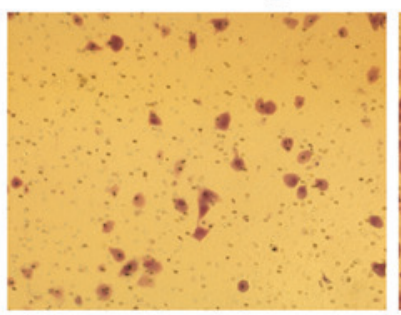

$5-8$ F/pEGFP-C1

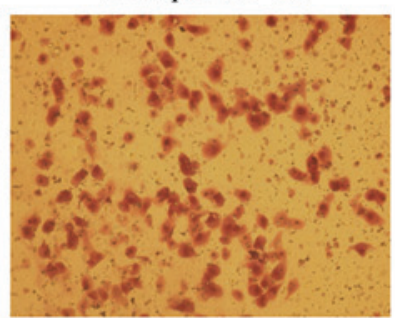

6-10B/siRNA-negative

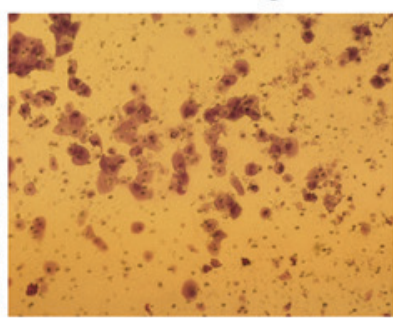

5-8F/pEGFP-C1-Annexin A1

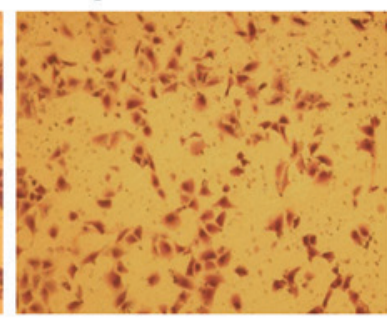

6-10B/siRNA-Annexin A1

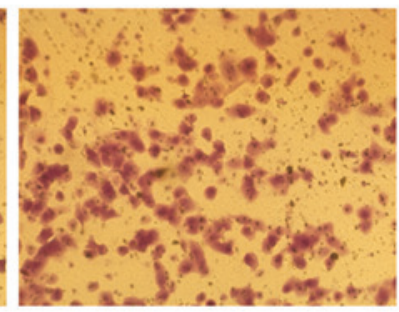

5-8F/pEGFP-C1-Annexin A1

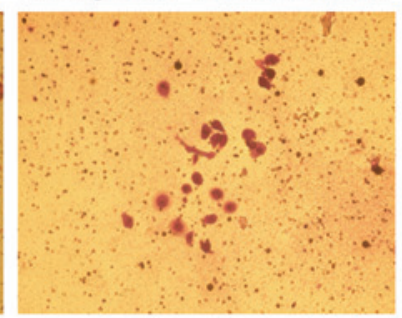

6-10B/siRNA-Annexin A1

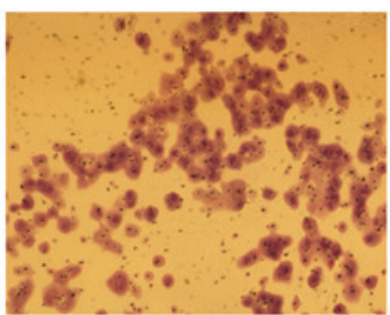

Figure 5. In vitro migration and invasion assays. The in vitro migration of (A) the $5-8 \mathrm{~F}$ cells and (B) the $6-10 \mathrm{~B}$ cells and their transfectants was measured using Transwell chambers. The in vitro invasion of (C) the 5-8F cells and (D) the 6-10B cells and their transfectants was measured using Transwell chambers. Original magnification, x100. siRNA, small interfering RNA; EGFP, enhanced green fluorescent protein.

\section{Discussion}

Annexin A1 is established as a multifunctional protein that is involved in inflammatory responses, cell differentiation and proliferation, regulation of cell death signals, phagocytic clearance of apoptotic cells and other biological activities in cells $(15,16)$. Previous studies have also demonstrated that Annexin A1 has significant inhibitory effects on cell growth and proliferation in lung (8) and larynx cancer (17). Annexin A1 has antiproliferative activity in macrophages due to the constitutive activation of the mitogen-activated protein kinase (MAPK)/extracellular signal-regulated kinase (ERK) pathway, which was linked to its phosphorylation by epidermal growth factor (EGF) (8). In squamous epithelial tumor types, Annexin A1 binds to calcium-dependent phospholipase A2 (cPLA2) by interacting with S100A11, inhibiting its activity, thus inhibiting the epidermal growth factor receptor (EGFR) signaling pathway (18). In addition, Annexin A1 also inhibits cell proliferation by another signal transduction pathway. Annexin A1 Ser residue (Section 27) is known to be the phosphorylation target for protein kinase $\mathrm{C}$ (PKC) and participates in PKC-mediated intracellular signal transduction. Annexin A1 is considered to have a src-homology 2 (SH2) domain and binds to the Grb-2 adaptor protein, which lies upstream of the MAPK pathway (8). Annexin A1 is considered to exert its antiproliferative activity via ERK-mediated disruption of the actin cytoskeleton and inhibition of cyclin D1, but not by induction of $\mathrm{p} 21^{\text {cip//waf1 } 1}(19,20)$. In one study, Annexin A1 expression was upregulated following transfection of an Annexin A1 viral expression vector into prostate cancer cells, which express low levels of Annexin A1. The results revealed that re-expression of Annexin A1 reduced the cell viability and cell colony 
formation and inhibited their proliferation (21). These data suggest Annexin A1 may inhibit the proliferation of tumor cells in various different manners.

To investigate the association of Annexin A1 expression with NPC proliferation and metastasis, the expression levels of Annexin A1 in NPC cell lines 5-8F and 6-10B with different metastatic potentials were detected by western blot analysis. The 5-8F and 6-10B cell lines originated from the NPC cell line SUNE-1 and have the same genetic background but different metastatic potentials, which are ideal for investigating the changes involved in tumor metastasis (22). The result of western blot analysis demonstrated that the expression levels of Annexin A1 were significantly lower in highly metastatic $5-8 \mathrm{~F}$ cells than those in non-metastatic 6-10B cells, suggesting that Annexin A1 downregulation may be associated with the increased metastatic potential of NPC cells. This is consistent with the results of our previous study $(10,14)$, indicating that the loss of Annexin A1 expression is associated with clinical NPC metastasis.

Then, an Annexin A1 overexpression vector and interference vector, and stably transfected $5-8 \mathrm{~F}$ and $6-10 \mathrm{~B}$ cells were constructed, respectively. By observing the cell behavior, it was identified that the upregulation of Annexin A1 in NPC cells significantly reduced cell proliferation and the number and volume of colony-forming cells, while the percentage of cells in G1 phase increased. Conversely, downregulation of Annexin A1 in NPC cells significantly accelerated cell proliferation, increased the number and volume of colony-forming cells and increased the percentage of cells in $\mathrm{S}$ phase. The results suggested that Annexin A1 expression may inhibit the tumorigenic processes of NPC.

Invasion is one of the key components of the metastatic cascade, so whether Annexin A1 expression levels are associated with the cell migration and invasion of NPC was examined in vitro. The results demonstrated that overexpression of Annexin A1 in 5-8F cells was able to decrease their in vitro migration and invasion ability, whereas downregulation of Annexin A1 expression in 6-10B cells increased their in vitro cell migration and invasion ability, which demonstrated that the expression levels of Annexin A1 were inversely associated with the in vitro migratory and invasive capability of NPC cells. Taken together, these data suggested that a decrease in Annexin A1 expression was associated with enhanced metastasis in NPC.

Other cancer studies have also demonstrated a correlation between the levels of Annexin A1 and tumor cell proliferation and metastasis. Paweletz et al (23) found that the loss of Annexin A1 was closely associated with the tumorigenesis of esophageal tissue and tumor differentiation. This study demonstrated that Annexin A1 was highly positive in normal esophageal epithelium mucosa by immunohistochemical staining, while in the malignant transformation of epithelial tissues, staining was significantly decreased, and in the highly invasive tumor tissue, Annexin A1 was absent. In several studies on breast cancer (24-26), it was revealed that the levels of Annexin A1 in normal breast epithelial, hyperplastic and breast cancer tissues decreased gradually, and that the degree of Annexin A1 expression deficiency was connected with tumor size and clinical staging. For example, if the clinical stage of NPC was higher and its differentiation was lower, the levels of Annexin A1 expression were lower. Babbin et al (27) also identified that in colorectal cancer, Annexin A1 has an important role in the process of metastasis of tumor cells. Another study (28) demonstrated that Annexin A1 was able to regulate the invasion and metastasis of gastric cancer cells, and affected the patient's prognosis directly both in vivo and in vitro.

In conclusion, the present study demonstrated that Annexin A1 may be a suppressor gene of NPC. However, few metastasis suppressors have been identified in NPC, and the mechanism through which they suppress metastasis remains elusive. Therefore, the identification of Annexin A1, whose expression was associated with the suppression of the growth and invasive ability of NPC cell lines, may contribute to the understanding of the development and progression of NPC, and provide an experimental basis for NPC targeted therapy.

\section{Acknowledgements}

This study was supported by grants from the National Natural Science Foundation of China (nos. 81072198, 81172210 and 81272959), the Scientific Research Fund of Hunan Provincial Education Department (no. 10A104), the National Natural Sciences Foundation of Hunan (no. 10JJ6035, 12JJ6080) and the Construct Program of the Key Discipline in Hunan Province (no. 2011-76).

\section{References}

1. Huang TR, Zhang SW, Chen WQ, et al: Trends in nasopharyngeal carcinoma mortality in China, 1973-2005. Asian Pac J Cancer Prev 13: 2495-2502, 2012.

2. Chan AT: Nasopharyngeal carcinoma. Ann Oncol 21 (Suppl 7): vii308-vii312, 2010.

3. Yu MC and Yuan JM: Epidemiology of nasopharyngeal carcinoma. Semin Cancer Biol 12: 421-429, 2002.

4. Ren JS, Chen WQ, Shin HR, et al: A comparison of two methods to estimate the cancer incidence and mortality burden in China in 2005. Asian Pac J Cancer Prev 11: 1587-1594, 2010.

5. Chang ET and Adami HO: The enigmatic epidemiology of nasopharyngeal carcinoma. Cancer Epidemiol Biomarkers Prev 15: 1765-1777, 2006.

6. King AD, Ahuja AT, Leung SF, et al: Neck node metastases from nasopharyngeal carcinoma: MR imaging of patterns of disease. Head Neck 22: 275-281, 2000.

7. Huang CJ, Leung SW, Lian SL, Wang CJ, Fang FM and Ho YH: Patterns of distant metastases in nasopharyngeal carcinoma. Kaohsiung J Med Sci 12: 229-234, 1996.

8. Lim LH and Pervaiz S: Annexin 1: the new face of an old molecule. FASEB J 21: 968-975, 2007.

9. Patel DM, Ahmad SF, Weiss DG, Gerke V and Kuznetsov SA: Annexin A1 is a new functional linker between actin filaments and phagosomes during phagocytosis. J Cell Sci 124: 578-588, 2011.

10. Garcia Pedrero JM,Fernandez MP, Morgan RO, et al: Annexin A1 down-regulation in head and neck cancer is associated with epithelial differentiation status. Am J Pathol 164: 73-79, 2004.

11. Zhang L, Yang X, Zhong LP, et al: Decreased expression of Annexin A1 correlates with pathologic differentiation grade in oral squamous cell carcinoma. J Oral Pathol Med 38: 362-370, 2009.

12. Wang LD, Yang YH, Liu Y, Song HT, Zhang LY and Li PL: Decreased expression of annexin A1 during the progression of cervical neoplasia. J Int Med Res 36: 665-672, 2008.

13. Chiu CC, Lin CY, Lee LY, et al: Molecular chaperones as a common set of proteins that regulate the invasion phenotype of head and neck cancer. Clin Cancer Res 17: 4629-4641, 2011.

14. Cheng AL, Huang WG, Chen ZC, et al: Identificating cathepsin $\mathrm{D}$ as a biomarker for differentiation and prognosis of nasopharyngeal carcinoma by laser capture microdissection and proteomic analysis. J Proteome Res 7: 2415-2426, 2008. 
15. Gerke V and Moss SE: Annexins: from structure to function. Physiol Rev 82: 331-371, 2002.

16. McArthur S, Cristante E, Paterno M, et al: Annexin A1: a central player in the anti-inflammatory and neuroprotective role of microglia. J Immunol 185: 6317-6328, 2010.

17. Lecona E, Barrasa JI, Olmo N, Llorente B, Turnay J and Lizarbe MA: Upregulation of annexin A1 expression by butyrate in human colon adenocarcinoma cells: role of p53, NF-Y, and p38 mitogen-activated protein kinase. Mol Cell Biol 28: 4665-4674, 2008 .

18. Sakaguchi M, Murata H, Sonegawa H, et al: Truncation of annexin A1 is a regulatory lever for linking epidermal growth factor signaling with cytosolic phospholipase A2 in normal and malignant squamous epithelial cells. J Biol Chem 282: 35679-35686, 2007.

19. Alldridge LC and Bryant CE: Annexin 1 regulates cell proliferation by disruption of cell morphology and inhibition of cyclin D1 expression through sustained activation of the ERK1/2 MAPK signal. Exp Cell Res 290: 93-107, 2003.

20. Parente L and Solito E: Annexin 1: more than an anti-phospholipase protein. Inflamm Res 53: 125-132, 2004.

21. Hsiang CH, Tunoda T, Whang YE, Tyson DR and Ornstein DK The impact of altered annexin I protein levels on apoptosis and signal transduction pathways in prostate cancer cells. Prostate 66: 1413-1424, 2006.
22. Yang XY, Ren CP, Wang L, et al: Identification of differentially expressed genes in metastatic and non-metastatic nasopharyngeal carcinoma cells by suppression subtractive hybridization. Cell Oncol 27: 215-223, 2005.

23. Paweletz CP, Ornstein DK, Roth MJ, et al: Loss of annexin 1 correlates with early onset of tumorigenesis in esophageal and prostate carcinoma. Cancer Res 60: 6293-6297, 2000.

24. Deng S, Wang J, Hou L, et al: Annexin A1, A2, A4 and A5 play important roles in breast cancer, pancreatic cancer and laryngeal carcinoma, alone and/or synergistically. Oncol Lett 5: 107-112, 2013.

25. Yom CK, Han W, Kim SW, et al: Clinical significance of annexin A1 expression in breast cancer. J Breast Cancer 14: 262-268, 2011.

26. Wang LP, Bi J, Yao C, et al: Annexin A1 expression and its prognostic significance in human breast cancer. Neoplasma 57: 253-259, 2010.

27. Babbin BA, Lee WY, Parkos CA, et al: Annexin I regulates SKCO-15 cell invasion by signaling through formyl peptide receptors. J Biol Chem 281: 19588-19599, 2006.

28. Cheng TY, Wu MS, Lin JT, et al: Annexin A1 is associated with gastric cancer survival and promotes gastric cancer cell invasiveness through the formyl peptide receptor/extracellular signal-regulated kinase/integrin beta-1-binding protein 1 pathway. Cancer 118: 5757-5767, 2012. 